\title{
Measurement of forearm rotation: The technique, and its reproducibility and validity
}

\author{
A. J. M. VAN DER VAART \\ Delft University of Technology, Delft, The Netherlands
}

\begin{abstract}
Forearm rotation is a point of interest in the study of human movement. For the most part, earlier methods for determining this joint rotation could be applied only in restricted situations. In this paper, a new technique is described, which involves the use of a three-dimensional opto-electronic motion registration system. With only two cameras, the supination angle in almost every position can be determined. A bracelet is attached to the subject's forearm and fixed to the radius. Wrist points are defined in relation to this bracelet (in its local coordinate system). The properties of the bracelet allow the motion-registration system to calculate its position in the global coordinate system. Given the position of the bracelet, the shoulder, and the elbow, the rotation angle can be determined. The reproducibility (error less than $2^{\circ}$ ) of and validity of the technique are also discussed.
\end{abstract}

Forearm rotations-supination 1 and pronation-are a point of interest in several areas of scientific study, such as neuroscience (Lacquaniti \& Soechting, 1982), orthopedics (Morrey, Askew, An, \& Chao, 1981), biomechanics (Langrana, 1981), and ergonomics (Marras \& Schoenmarklin, 1993). Up to now, many methods have been developed for registering forearm rotations; these methods include the use of visual observation (Davis, 1977), ordinary goniometers (Boone \& Azen, 1979), gravity goniometers (Laupattarakasem, Sirichativapee, Kowsuwon, Sribunditkul, \& Suibnugarn, 1990), electrogoniometers (Chao, 1980), biplanar videotaping (Langrana, 1981), and three-dimensional (3-D) measurement (Safaee-Rad, Shwedyk, \& Quanbury, 1987). The objective of the present study is to describe a new, accurate (with error of less than $2^{\circ}$ ), and valid method for assessing the forearm rotation angle.

Because of advances in technology, methods for determining this angle have gradually become more precise and more applicable. Nowadays, special motionregistration systems are available for determining human motion accurately. A major problem, however, with most of the opto-electronic motion-registration systems is the use of markers that fall outside the view of the cameras. Thus the 3-D positions of these markers are lost, and joint rotation cannot be continuously determined. When one is assessing forearm rotations, it is impossible to find a position in space that would allow an observer to see a point on the wrist for every supination angle at any time.

Correspondence should be addressed to A. J. M. van der Vaart, Faculty of Industrial Design Engineering, Delft University of Technology, Jaffalaan 9, 2628 BX Delft, The Netherlands (e-mail: a.j.m.vandervaart @io.tudelft.nl).
Since it is necessary to have at least two wrist points in constant view of the cameras, these systems can only be used in limited situations.

An easy way to solve the problem of the disappearing marker would be to use more cameras, so that each marker would be in constant view of the cameras. Since this solution would require a major investment, we have provided another. Our solution consists of a rigid wrist bracelet in which wrist points are defined locally. When the position of the bracelet in the global coordinate system is known, the wrist point positions, which are defined in the local coordinate system of the bracelet, can be determined, and thus the supination angle can be calculated.

In the present report, we describe this new technique; we also discuss the reproducibility of its results, and validation of its assumptions.

\section{Definition of Supination}

Here the term supination (see Joseph, 1982, p. 370) will refer to the rotation of the radius in the distal and proximal radioulnar joints, resulting in a local rotation along the axis that is fixed relative to the ulna (Figure 1).

\section{Mathematical Basis}

If the arm is modeled by three vectors (Figure 2) consisting of (1) the upper arm (ua), from the shoulder joint to the elbow, (2) the forearm (fa), from the elbow to the ulnar side of the wrist, and (3) the wrist (wr), from the ulnar side to the radial side of the wrist, the supination angle can be determined.

If fa coincides with the supination axis, the supination angle $(\phi)$ will be the angle between the wrist vector (wr) and its projection (wr') on the arm plane, defined by ua 


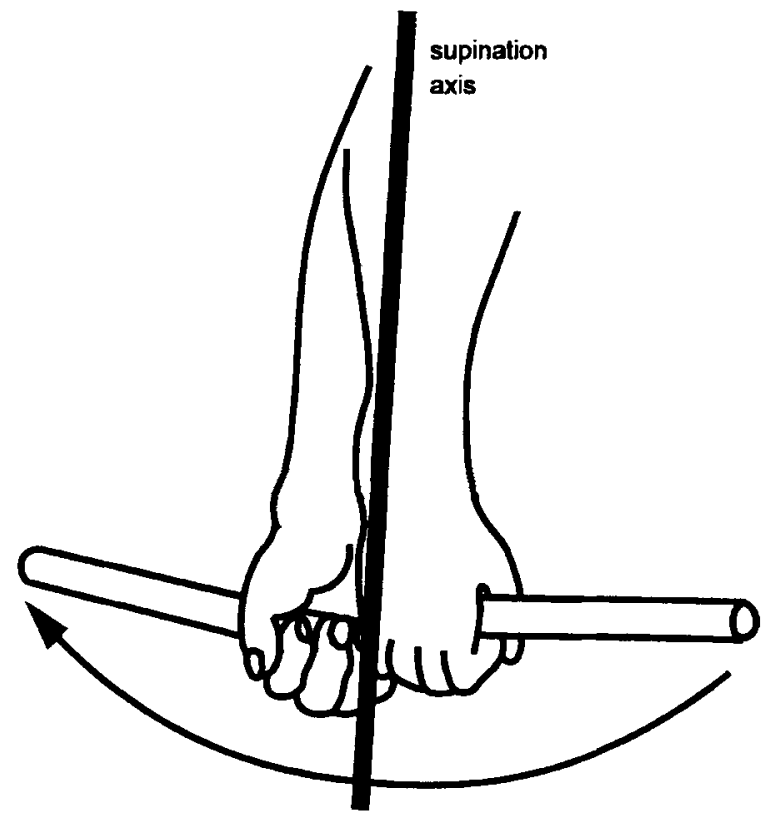

Figure 1. Forearm rotation (i.e., supination), from minimal supination (or maximal pronation) to maximal supination (or minimal pronation).

and fa. Given the 3-D vectors to the shoulder (s), the elbow (e), and the two wrist points $\left(\mathbf{w}_{1}, \mathbf{w}_{2}\right)$, the vectors can be determined:

$$
\begin{aligned}
\mathbf{u a} & =\mathbf{e}-\mathbf{s} \\
\mathbf{f a} & =\mathbf{w}_{1}-\mathbf{e} \\
\mathbf{w r} & =\mathbf{w}_{2}-\mathbf{w}_{1} ;
\end{aligned}
$$

and the supination angle can be calculated by

$$
\phi=90-\arccos \left[\frac{(\mathbf{u a} \times \mathbf{f a}) \cdot \mathbf{w r}}{|\mathbf{u a} \times \mathbf{f a}||\mathbf{w r}|}\right] .
$$

The supination angle $(\phi)$ will be zero when the distal end of the radius $\left(\mathbf{w}_{2}\right)$ is located in the plane formed by the upper arm and the distal end of the ulna $\left(\mathbf{w}_{1}\right)$ - that is, when the thumb is "up" and in the plane defined by the two arm segments. This method implies that the supination angle cannot be determined when the elbow angle is fully extended.

\section{TECHNIQUE}

\section{Motion Registration System}

The system used is the Optotrak 2010 (Northern Digital Inc., Waterloo, ON, Canada) 3-D opto-electronic motion registration system. This system uses "active" markers, meaning that the markers, placed on a subject, are controlled by the system. The markers radiate infrared light (from infrared-emitting diodes, or IREDs) that can be detected by cameras. With the aid of two cameras the system is able to calculate 3-D positions of the markers. The 3-D distance error is less than $0.5 \mathrm{~mm}$ at a camera distance of $4 \mathrm{~m}$. To assess the motion of an object, IREDs have to be fixed onto it. The 3-D positions of these markers are sampled by the system, at a chosen sample frequency. As a consequence, the motion is divided into several consecutive frames, each containing the position of the markers in one sample.

The minimum system, under which the Optotrak 2010 works well, consists of an IBM-compatible personal computer (Intel 286 processor or higher) with a minimum $640 \mathrm{~K}$ of RAM. The maximum sample frequency is directly coupled to the number of markers used: the number of markers times the installed sample frequency must be less than the maximum sample rate $(2500 \mathrm{~Hz})$.

\section{Marker Positions}

Because of the definition of supination used here, the most correct positions of the markers will be on the rotation centers within the joints. The shoulder marker should ideally be placed at the rotation center of the head of the humerus; the elbow marker should be on the junction of the elbow flexion axis, the longitudinal rotation axis of the humerus, and the supination axis; the first wrist marker should be on the supination axis at the head of the ulna; and the second wrist marker, somewhere on the distal part of the radius. Since it is not possible to fasten the markers directly onto bony landmarks or onto the rotation centers, the shoulder marker is fixed above the major tubercle of the upper arm (see Figure 3a), and the second marker is placed on the elbow above the lateral epicondyle of the upper arm (see Figure 3b).

Because the wrist markers will frequently disappear from the cameras, the two wrist points are defined in the

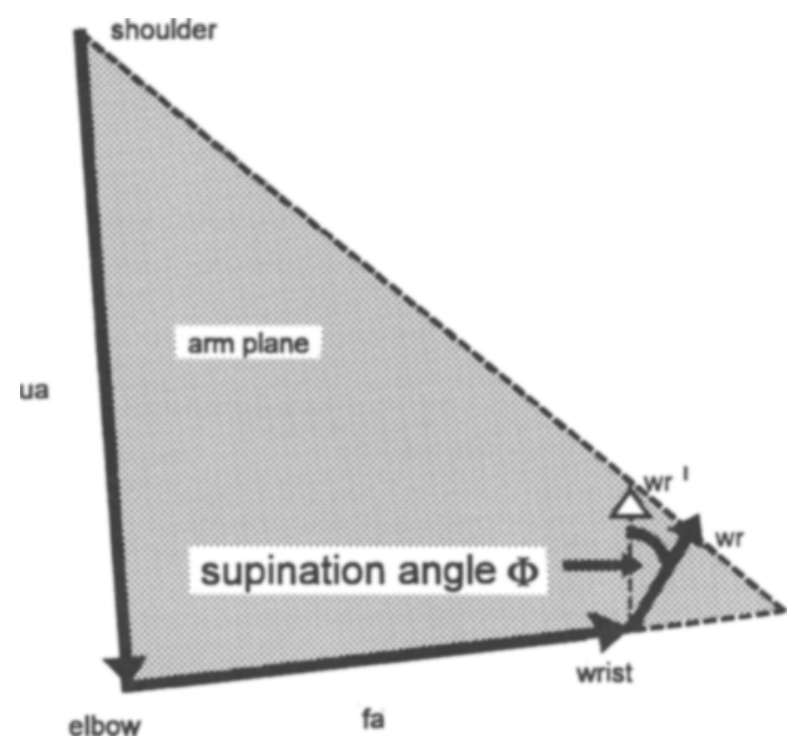

Figure 2. Vectorial representation of the arm and supination angle. 

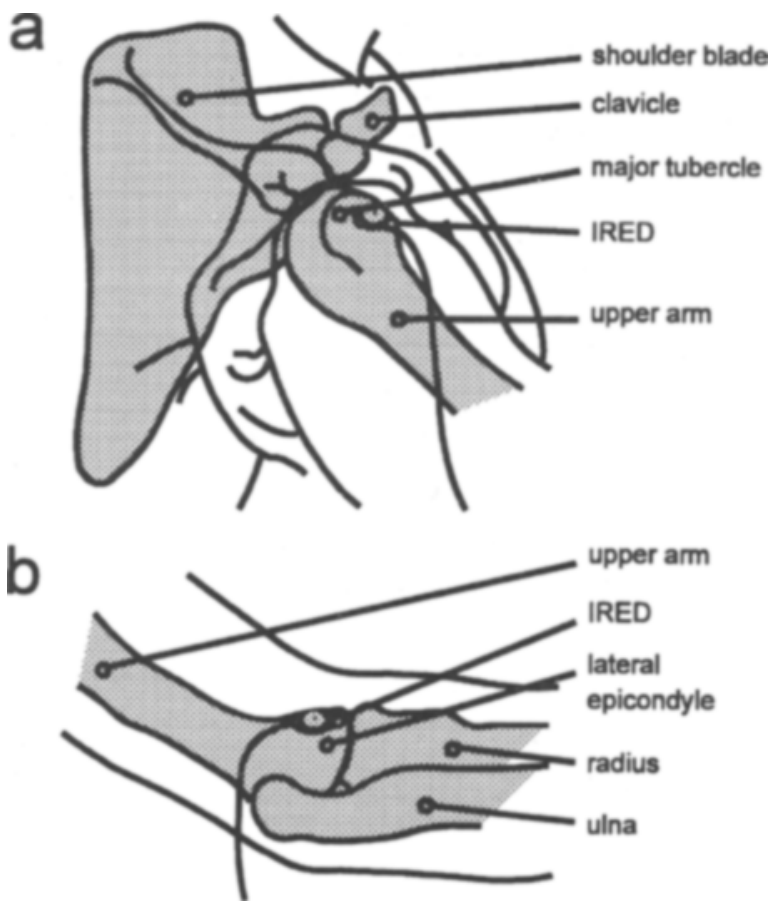

Figure 3. The position of the shoulder and elbow marker relative to bony landmarks. (a) The position of the shoulder marker above the major tubercle of the upper arm. (b) The position of the elbow marker at the lateral epicondyle of the upper arm.

local coordinate system of a wrist bracelet, which is located on the distal part of the forearm (see below), and whose position in the global coordinate system can always be determined. An easy way to define the two wrist points is to place the first wrist point at the center of the bracelet. However, because of the distance from the first wrist point to the actual supination axis, this will result in an increased inadequacy of the method (up to $7^{\circ}$; see under Reproducibility, below). Therefore, we chose to place the first wrist point onto the supination axis as accurately as possible. The second wrist point is defined on the distal part of the radius.

\section{Wrist Bracelet}

The bracelet (see Figure 4) consists of an aluminum ring; its outer diameter is $100 \mathrm{~mm}$, the inner diameter is $90 \mathrm{~mm}$, its width is $25 \mathrm{~mm}$, and its weight $150 \mathrm{~g}$. To fix the bracelet onto the distal part of the radius, an inflatable rubber tube is used as a cuff inside the bracelet. The internal pressure of the tube, needed to fix the bracelet onto the arm, is far less than the diastolic blood pressure, indicating that no occlusion of blood flow will be caused. On the outside of the bracelet 18 equal facets have been made at successive angles of $20^{\circ}$ to each other. The valve of the tube is put through one facet, while on the 17 other facets IREDs are glued. In this way, at least three markers will always be in view of both cameras.
The vector, in the local coordinate system of the bracelet, to each IRED $\left(\mathbf{b}_{i_{0}}=1, \ldots 17, n=0\right.$, indicating the local coordinate system), is known.

The bracelet has to be fixed onto the radius, but not onto the ulna. To avoid the influence of any disturbing motions of the head of the ulna on the position of the bracelet relative to the radius, a stiff aluminum plate is wrapped around the head of the ulna, between the tube and the skin (see Figure 5). The tube is then fixed onto the radius and the aluminum plate. Consequently, the head of the ulna has no influence on the fixation of the bracelet onto the radius.

\section{Supination Axis}

The position of the two wrist points in the local coordinate system of the bracelet can be determined by the following procedure. First the supination axis is determined. Next, this axis is intersected with a plane defined on the bracelet. This intersection point is defined as the earlier mentioned first wrist point, described by $\mathbf{w}_{1}$. The second wrist point, described by $\mathbf{w}_{2}$, is defined at a distance of $50 \mathrm{~mm}$ from the first wrist point, also in the local coordinate system of the bracelet. Consequently, this point is defined in the same local coordinate system, located on the distal part of the radius, as is the first wrist point. To be able to define the position of the first wrist point in the local coordinate system of the bracelet, the position of the supination axis has to be known.

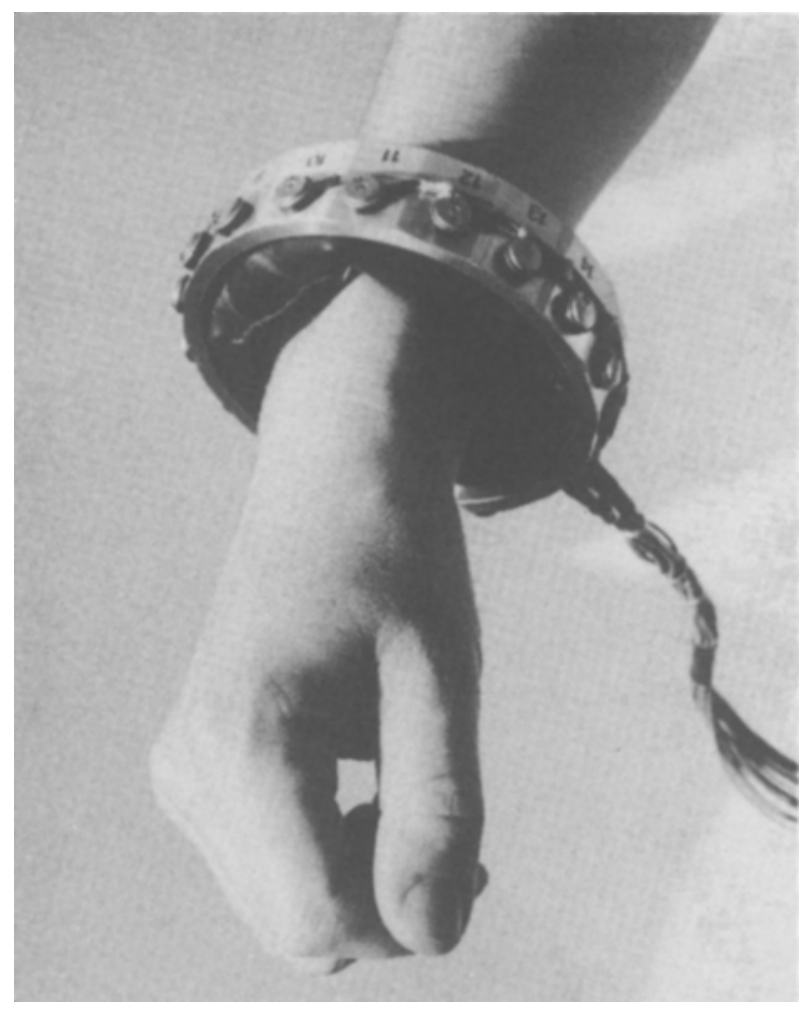

Figure 4. The wrist bracelet fixed on the distal part of the forearm. 


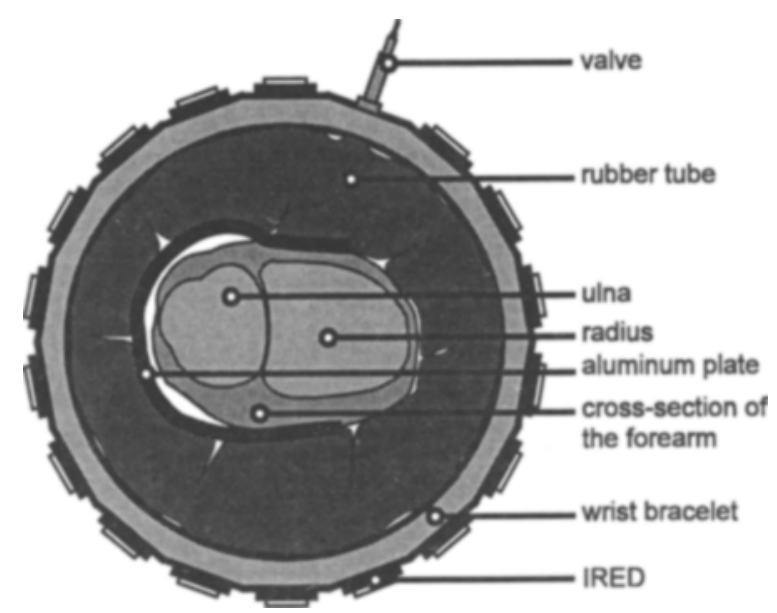

Figure 5. Transversal cross-section of the wrist bracelet fixed on the forearm. The aluminum plate permits the bracelet to be fixed only onto the radius, not the ulna.

As mentioned above, the supination axis is defined as the axis around which the radius rotates in relation to the ulna. To determine the position of this supination axis, a special trial is needed. In this trial, the supination axis is determined in the global coordinate system, because it is not possible to do this in the local coordinate system of the ulna. When, however, the position of the ulna remains steady, the global coordinate system can substitute for the local coordinate system. Therefore, the subject has to sit in a chair and put his/her arm on an armrest, on which a block with a longitudinal groove is placed. The upper arm hangs down; the elbow is flexed $90^{\circ}$. The subject has to place the ulna into the groove of the block. In this situation he/she can perform a pure pronation and supination by using tactile information; the ulna is not moved relative to the block, i.e. the global coordinate system.

In the initial position, the subject has to hold his/her hand in a sagittal plane, with the thumb pointing upward. The task is to perform a pronation followed by a supination. Neither rotation is allowed to be maximal, because maximal forearm rotations often induce ulnar motions relative to the block. The total range of the rotation is therefore required to be about $90^{\circ}$. This motion is sampled by the system to determine the rotation axisthe supination axis - of the bracelet.

In this trial, the transformation of the bracelet is due only to a rotation around the supination axis. One method for describing the change of position of the bracelet is to use a helical axis. If an object is considered rigid and its movement between two subsequent positions is taken to be a screw motion, such a motion can be described by the position of the screw or helical axis, the angle of rotation about this axis, and the translation along this axis (Spoor \& Veldpaus, 1980). In case of a transformation that only consists of a rotation, the heli- cal axis will be the same as the rotation axis; that is, the translation component along the helical axis is zero. As in the situation described above, the change of position of the wrist bracelet is due only to the rotation of the radius relative to the ulna; for supination, no translation component is expected, and the helical axis will be the same as the supination axis. The method is at its most exact when the two positions, needed to determine the helical axis, are at an angle of about $90^{\circ}$ to each other. Therefore, the two outer positions of pronation and supination are used to determine the helical axis.

Spoor and Veldpaus (1980) have described a method for calculating the helical axis by using spatial coordinates of markers. The method consists of two parts. The first concerns the determination of the rotation matrix and the translational vector, and the second, the helical axis.

In our method, the first part is carried out by the program RIGID (Northern Digital Inc.). This program calculates for each frame $(n)$ the transformation of a predefined rigid body of the bracelet from the local coordinate system $(n=0)$ to the global coordinate system:

$$
\mathbf{b}_{i n}=\mathbf{R}_{n} \mathbf{b}_{i_{0}}+\mathbf{T}_{n} . \quad(i=1, \ldots 17)
$$

The output of this program consists, for each frame $(n)$, of a rotation matrix $\left(\mathbf{R}_{n}\right)$ and a translation vector $\left(T_{n}\right)$. The rotation matrix and translation vector, which are needed as input for the second part, must, however, describe the transformation between two subsequent positions $(n$ and $n+1)$. These can be easily calculated as

$$
\begin{aligned}
\mathbf{R}_{n \rightarrow n+1} & =\mathbf{R}_{n+1} \mathbf{R}_{n}^{-1} \\
\mathbf{T}_{n \rightarrow n+1} & =\mathbf{T}_{n+1}-\mathbf{R}_{n \rightarrow n+1} \mathbf{T}_{n} .
\end{aligned}
$$

The rotation matrix and translational vector are used as the input for the second part of the method of Spoor and Veldpaus (1980). The outcome of the second part is the helical axis-that is, the supination axis.

\section{Wrist Points}

As described earlier, the vector to the first wrist point $\left(\mathbf{w}_{1_{0}}\right)$, in the local coordinate system of the bracelet $(n=0)$, will be defined by the intersection point of the supination axis and the plane of the bracelet. The frame used to determine the position of the plane of the bracelet is taken from the start of the trial $(n=1)$. The plane of the bracelet, in the global coordinate system, can be determined by using the bracelet vectors $\mathbf{b}_{1}, \mathbf{b}_{7_{1}}$, and $\mathbf{b}_{13_{1}}$ (three equidistant markers of the bracelet; see Figure 6).

With the output of the program RIGID, the actual positions of the three needed bracelet IREDs are determined (Equation 3 ). The vector to the intersection point, $\mathbf{w}_{1_{1}}$, can be described with the vectors $\left(\mathbf{b}_{7_{1}}-\mathbf{b}_{1_{1}}\right)$ and $\left(\mathbf{b}_{13_{1}}-\mathbf{b}_{1_{1}}\right)$. The contribution of each of these two vec- 


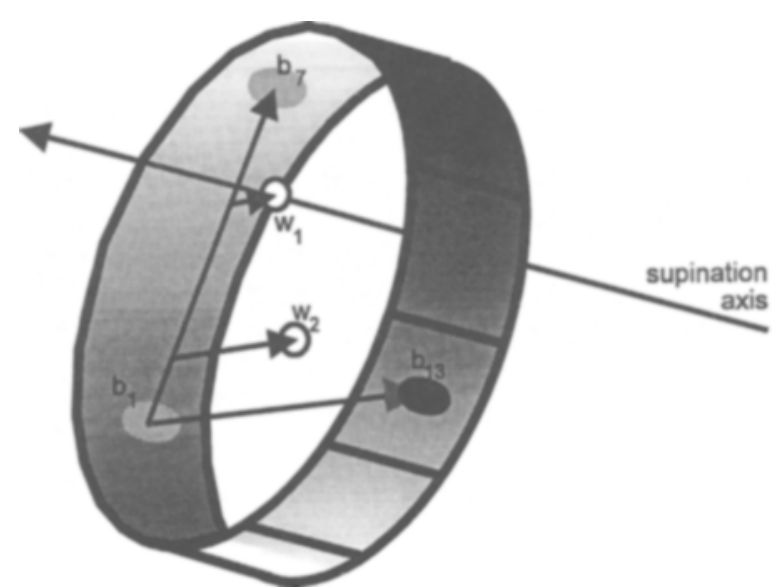

Figure 6. The representation of the two wrist points $\left(w_{1}\right.$ and $\left.w_{2}\right)$ in the local coordinate system of the bracelet.

tors is given by the scalars $\lambda_{1}$ and $\mu_{1}$. The second wrist point is also defined in the plane of the bracelet, above the first wrist point. This wrist point can be locally described with the scalars $\lambda_{2}$ and $\mu_{2}$.

With the parameters $\lambda_{i}$ and $\mu_{i}(i=1,2)$ known and the knowledge of the actual position of the three bracelet IREDs in a specific frame, the two wrist points can be determined in that frame $(n)$ :

$$
\mathbf{w}_{i_{n}}=\mathbf{b}_{1_{n}}+\lambda_{i}\left(\mathbf{b}_{7_{n}}-\mathbf{b}_{1_{n}}\right)+\mu_{i}\left(\mathbf{b}_{13_{n}}-\mathbf{b}_{1_{n}}\right),(i=1,2)
$$

which solves Equation 1, and consequently the supination angle $(\phi)$ in Equation 2.

\section{Calibration}

The neutral position of supination is defined as the initial position of the forearm in the special trial: the hand in a sagittal plane and the thumb pointing upward. In this position the supination angle is defined as $\phi=0$.

At the start of this trial, the two wrist points are defined. When calculating the supination angle, the positions of the shoulder and the elbow also have to be taken into consideration (see Equation 2). The two markers, indicating the position of the shoulder and elbow, are

Table 1

Position of the Rotation Point of the Bracelet in the Local Coordinate System $(x, y$ in Millimeters)

\begin{tabular}{|c|c|c|}
\hline Trial & $x$ & $y$ \\
\hline 1 & 55.7 & -11.8 \\
\hline 2 & 56.9 & -11.4 \\
\hline 3 & 56.9 & -13.1 \\
\hline 4 & 57.6 & -9.2 \\
\hline 5 & 58.3 & -11.6 \\
\hline RMS & & \\
\hline
\end{tabular}

Note-The rotation point was determined five times on the forearm of 1 subject. The RMS value is the root mean square of the differences between the individual rotation points and the mean rotation point. fixed onto the outside of the body. Consequently, due to the skin and muscles, these marker positions deviate from the actual desired positions. In all cases this will result in a systematic difference between the calculated and defined supination angle. To correct for such a deviation, the initial position of the special trial (controlled by the experimenter) is used as calibration. In this trial, the supination angle is defined as zero. The initially calculated supination angle, insofar as it differs from zero, can be seen as the systematic deviation. Therefore, for each assessment of supination, the initially calculated value is subtracted.

\section{REPRODUCIBILITY AND VALIDITY OF THE MEASUREMENT OUTCOMES}

\section{Reproducibility}

The reproducibility of the supination angle will depend mainly on the reproducibility of the first wrist point. To determine the reproducibility of the first wrist point, 1 subject was asked to perform the special rotation task five times. Within these trials, the position of the bracelet, relative to the forearm, was not altered. Therefore, the position of the first wrist point, in the local coordinate system, was expected to be constant. Table 1 shows for each trial the $x$ and $y$ coordinates of the first wrist point in the local coordinate system of the bracelet. The root mean square of the distances of these points, relative to the mean rotation point, was $1.5 \mathrm{~mm}$.

This position error, within the wrist point determination, will influence the outcomes of the supination calculations. The position error of the second wrist point is by definition the same as the position error of the first wrist point. Since supination is the angle between the wrist vector and the projection of it on the arm plane (Equation 2), the consequence of these errors on the outcomes of the supination calculation will depend on the flexion angle of the elbow: owing to the position error of the wrist points, the position of the wrist vector as well as the orientation of the arm plane will be influenced. In the case of a flexion of $90^{\circ}$, there will be no influence, but when the elbow is nearly extended, the influence will be greater. When the position of the wrist points is changed within a distance of $1.5 \mathrm{~mm}$, and the elbow angle is changed within a range of $10^{\circ}$ to $90^{\circ}$, this error is less than $2^{\circ}$. Therefore, it can be concluded that the supination angle can be reproduced within a range of $2^{\circ}$.

In a small pilot experiment, the effect of the correctness of the position of the first wrist point was worked out. It was found that when the first wrist point was simply defined to be in the center of the bracelet (which makes the method of determining the position of the supination axis superfluous), the difference between this method and the method described above (i.e., the first wrist point onto the supination axis) could be as much as $7^{\circ}$. The size of this difference depends on the 
size of the wrist: the bigger the wrist, the larger the difference. Moreover, the size of this difference depends on the actual size of the supination angle, which makes it difficult to correct.

\section{Validity}

The method is based on two assumptions that may turn out to be invalid: (1) the translation component of the helical axis is zero; and (2) supination takes place around one fixed axis.

Zero translation component. With the use of the helical axis as an estimate of the joint rotation axis, the translation component along the helical axis was assumed to be zero. This assumption was demonstrated to hold if the bracelet was fixed onto the stiff axle. In five independent trials, the mean translation component was only $-0.10 \mathrm{~mm}$, with a standard deviation of $0.14 \mathrm{~mm}$. Subsequently, 5 subjects were asked to perform the trial in which the helical axis was determined. The mean translation component was $-0.3 \mathrm{~mm}$, with a standard deviation of $1.5 \mathrm{~mm}$. The comparison of this outcome with the accuracy of the Optotrak system $(0.5 \mathrm{~mm})$ led to the conclusion that the translation component was negligible.

One fixed axis. In the technique described here, only one locally fixed supination axis is assumed. If this is not correct, the calculation of the wrist points, and therefore also the supination angle, will yield erroneous results. Since the forearm rotates in two distinct jointsthe proximal and distal radioulnar joints - it is expected that the rotation axis is fixed in relation to the ulna. However, the size of the contact area in both joints depends on the supination angle (Hagert, 1992), which indicates that both radioulnar joints are not ideal hinge joints. Theoretically, this will result in a changing position of the supination axis during supination.

When the bracelet was attached to the stiff axle, the fluctuation within one trial of the rotation point was determined. Between each two subsequent frames, the location of the rotation point of the wrist bracelet was calculated. Since the axle remained fixed in relation to the global coordinate system, this point was expected to be constant in the global coordinate system. The root mean square of the differences between the calculated 3-D position and mean assessed 3-D position was $0.4 \mathrm{~mm}$. In comparing this with the accuracy of the Optotrak system (less than $0.5 \mathrm{~mm}$ ) it is concluded that, in case of an axle with ball bearings, the assumption holds true.

To see whether the assumption of one fixed axis would hold in an experimental situation with subjects, the following experiment was carried out. In a trial with ulna fixed into the groove of the block, 6 subjects performed a forearm rotation. The positions of the IREDs were collected at a sample frequency of $25 \mathrm{~Hz}^{2}$ In the range between the outer pronation and outer supination positions, the supination axis was determined between each two subsequent frames. In addition, a helical axis was determined by using only the two outer positions (resulting in the supination axis used in the calculations). Each axis, when intersected with the plane of the bracelet, would result in a locally defined wrist point. In the case of a constant supination axis relative to the ulna, this position would not deviate from the point calculated by using only the two outer positions. The root mean square of the distance between the continuously determined and the used local wrist point was, respectively for each subject, $0.7,1.3,1.3,1.4,1.7$, and $1.9 \mathrm{~mm}$.

These values are all greater than $0.5 \mathrm{~mm}$ (i.e., the accuracy of the system). Therefore, it must be concluded that the helical axis changed position during the forearm rotation, and consequently the second assumption should be invalidated. But, since the root mean square values, compared with the reproducibility of the rotation point, were within an acceptable range, it may be concluded that the assumption of one fixed axis is reasonable and therefore could be used in the method.

\section{CONCLUSION}

The method reported here enables the determination of the supination angle through the use of an optoelectronic motion registration system. For the determination of the supination angle, at least four marker positions are needed: one on the shoulder, one on the elbow, and two on the wrist. During supination, the two wrist points frequently disappear out of the view of the cameras. To assess the position of these wrist points in the global coordinate system, a bracelet is used, and the two wrist points are defined in relation to this bracelet. The positions of these wrist points are reproducible within a distance of $1.5 \mathrm{~mm}$ in the local coordinate system of the bracelet. When the positions of the shoulder marker and the elbow marker and the global position of the bracelet are known, the supination angle can be calculated. Compared with the purchase of more cameras, the investment required is low. The results of this method have proved to be reproducible within two degrees of arc and are valid.

\section{REFERENCES}

Boone, D. C., \& AzEN, S. P. (1979). Normal range of motion of joints in male subjects. Journal of Bone \& Joint Surgery, 61A, 756-759.

Chao, E. Y. S. (1980). Justification of triaxial goniometer for the measurement of joint rotation. Journal of Biomechanics, 13, 989-1006.

DAvis, P. R. (1977). Some significant aspects of normal upper limb functions. In Joint replacement in the upper limb (pp. 1-5). London: Institute of Mechanical Engineers.

HAGERT, C. G. (1992). The distal radioulnar joint in relation to the whole forearm. Clinical Orthopaedics \& Related Research, 275, 56-64.

JOSEPH, J. (1982). A textbook of regional anatomy. London: MacMillan.

LaCQuaniti, F., \& Soechting, J. F. (1982). Coordination of arm and wrist motion during a reaching task. Journal of Neuroscience, 2, 399-408. 
LANGRANA, N. A. (1981). Spatial kinematic analysis of the upper extremity using a biplanar videotaping method. Journal of Biomechanical Engineering, 103, 11-17.

Laupattarakasem, W., Sirichativapee, W., Kowsuwon, W., SRibunditKul, S., \& Suibnugarn, C. (1990). Axial rotation gravity goniometer: A simple design of instrument and a controlled reliability study. Clinical Orthopaedics \& Related Research, 251, 271-274.

MarRas, W. S., \& SChoenMarkLIN, R. W. (1993). Wrist motion in industry. Ergonomics, 36, 341-351.

Morrey, B. F., Askew, L. J., AN, K. N., \& Chao, E. Y. (1981). A biomechanical study of normal functional motion. Journal of Bone \& Joint Surgery, 63A, 872-877.

Safaee-Rad, R., Shwedyk, E., \& Quanbury, A. O. (1987). Functional human arm motion study with a new 3-D measurement system (VCR-PIPEZ-PC). In Proceedings of IEEE-EMBS, 9th annual conference (pp. 54-56).

SPOOR, C. W., \& VeldPaUs, F. E. (1980). Rigid body motion calculated from spatial co-ordinates of markers. Journal of Biomechanics, 13, 391-393.

\section{NOTES}

1. Whenever supination is mentioned, it can mean supination as well as pronation (i.e., negative supination).

2 . Since the number of markers used is $19-17$ on the bracelet and 2 on the arm - the maximal sample frequency could be about $125 \mathrm{~Hz}$, or 2500/19. In the newest Optotrak system (Version 3020), the maxi- mum sample rate is $3500 \mathrm{~Hz}$, which will result in a maximum sample frequency of about $180 \mathrm{~Hz}$.

\section{APPENDIX \\ Nomenclature}

$\mathbf{b}_{i_{n}} i$ th marker of the bracelet in the $n$th frame

e vector to elbow

fa vector of the forearm

$i \quad$ number of marker

$n \quad$ frame number (when $n=0$, this refers to a local coordinate system)

$\mathbf{R}_{n}$ rotation matrix in $n$th frame

s vector to shoulder

$\mathbf{T}_{n}$ translation in $n$th frame

ua vector of the upper arm

$\mathbf{w}_{1_{n}}$ vector to ulnar wrist point in the $n$th frame

$\mathbf{w}_{2_{n}}$ vector to radial wrist point in the $n$th frame

wr vector of the wrist

$\lambda_{i} \quad$ wrist parameter of the $i$ th wrist point

$\mu_{i} \quad$ wrist parameter of the $i$ th wrist point

$\phi \quad$ supination angle 